\title{
Comunicação médico-paciente em ambulatórios de pediatria de um hospital universitário
}

\author{
Physician-patient communication in at outpatient of pediatrics \\ university hospital
}

Haline G. Lira', Camila V. B. Machado', leda R. L. Del Ciampo², Luiz A. Del Ciampo³

\begin{abstract}
RESUMO
Introdução: a relação médico-paciente depende de fatores relacionados aos indivíduos e às condições sociais e culturais que propiciam o encontro desses dois personagens. Objetivo: analisar alguns aspectos relacionados ao atendimento de crianças que frequentam os ambulatórios do HCFMRPUSP, no que diz respeito ao contato e ao diálogo com o corpo clínico.

Metodologia: estudo retrospectivo, observacional e descritivo desenvolvido nos ambulatórios do Hospital das Clínicas da Faculdade de Medicina de Ribeirão Preto - USP, por meio de entrevistas com as mães de crianças que aguardavam atendimento médico. Foi utilizado um questionário com perguntas relativas à criança (idade, sexo, tempo de acompanhamento no serviço), à mãe (idade, grau de escolaridade e situação conjugal) e à consulta (relacionamento com o médico, entendimento das orientações, aspectos positivos e negativos do atendimento e sugestões). O estudo foi aprovado pelo Comitê de Ética em Pesquisa do Hospital das Clínicas da Faculdade de Medicina de Ribeirão Preto da Universidade de São Paulo. Resultados: participaram 118 mães com mediana de idade foi de 32 anos, sendo $53,3 \%$ casadas e $22,8 \%$ solteiras. Entre as crianças, $57,6 \%$ eram do sexo feminino e $42,3 \%$ masculino. A mediana de escolaridade das mães apontou o segundo grau completo e o tempo médio de seguimento nos ambulatórios foi de 2 anos e 1 mês. 50\% das mães referiram que a terminologia técnica era a principal causa de dificuldade de entendimento durante a consulta, $34,7 \%$ destacaram a atenção dispensada pelo médico como o fator mais positivo, enquanto que $57,6 \%$ destacaram que a demora era o principal fator negativo durante o atendimento. Conclusões: as dificuldades de comunicação entre médico e paciente e o acoIhimento foram aspectos considerados importantes no atendimento oferecido pelo hospital universitário, devendo fazer parte do contexto dos profissionais que nele atuam.
\end{abstract}

Palavras-chave: Cuidados Médicos. Serviços de Saúde. Educação Médica. Satisfação do Paciente.

1. Aluno, 2. Médico Assistente, 3. Docente. Departamento de Puericultura e Pediatria da Faculdade de Medicina de Ribeirão Preto da Universidade de São Paulo.

Conflitos de interesse: não há
Correspondência Luiz Antonio Del Ciampo Avenida Bandeirantes, 3900 CEP. 14049-900 / Ribeirão Preto - São Paulo delciamp@fmrp.usp.br

Artigo recebido em 05/08/2014 Aprovado para publicação em 19/12/2014 


\begin{abstract}
Introduction: the doctor-patient relationship depends on factors related to individuals and their social and cultural conditions that enable the meeting of these two characters. Objective: to examine some aspects related to the care of children attending the outpatient clinics of the university hospital, with respect to the contact and dialogue with the clinical staff.

Methodology: retrospective, observational and descriptive study developed in the clinics of the Hospital das Clínicas, Faculty of Medicine of Ribeirão Preto - USP, through interviews with mothers of children awaiting medical care. We used a questionnaire with questions concerning to child (age, gender, followup time in service), to mother (age, educational level and marital status) and to relationship with the physician, understanding the guidelines, positives and negative attention and suggestions. The study was approved by the Research Ethics Committee of the Hospital of the Faculty of Medicine of Ribeirão Preto, University of São Paulo. Results: 118 mothers participated median age was 32 years, 53.3\% married and $22.8 \%$ unmarried. Among children, 57.6\% were female and $42.3 \%$ male. The median educational level of the mothers indicated a high school degree and the mean follow-up in outpatient clinics was 2 years and 1 month. $50 \%$ of mothers reported that the technical terminology was the main cause of the difficulty of understanding during the consultation, 34.7\% highlighted the attention given by the doctor as the biggest positive, while $57,6 \%$ pointed out that the delay was the main negative factor during service. Conclusions: the difficulties of communication between doctor and patient and the host were considered important in the care offered by the university hospital, and should be part of the working professional context.
\end{abstract}

Keywords: Medical Care. Health Services. Education, Medical . Patient Satisfaction.

\section{Introdução}

A prática médica, embora seja caracterizada pelo trabalho técnico e profissional, está intimamente ligada ao contato interpessoal. Mais que uma relação médico-paciente, estabelece um verdadeiro contrato de confiança e dependência entre um indivíduo que necessita de atenção e cuidados e outro que detém o conhecimento científico e as habilidades. ${ }^{1}$ Um dos aspectos mais relevantes dessa prática é o início do contato entre as partes, ou seja, como o diálogo é iniciado e estabelecido, de modo a facilitar todo o desenrolar da consulta e do acompanhamento médico. ${ }^{2,3,4}$

A relação médico-paciente estabelece-se de diversas maneiras, consolida-se com o tempo e depende de vários fatores relacionados ao paciente, ao médico e às condições sociais e culturais que propiciam o encontro desses dois personagens. Do ponto de vista acadêmico, a história clínica procura sistematizar a obtenção de informações que o médico necessita para iniciar a formulação de hipóteses diagnósticas que poderão ser confirmadas com o exame físico e procedimentos complementares. ${ }^{5,6,7}$ Nesse momento ocorre a interação entre o conhecimento do indivíduo sobre sua vida e o conhecimento teórico do médico calcado no processo saúde-doença, que deve culminar com a elaboração de hipóteses diagnósticas seguidas de um plano terapêutico. ${ }^{8}$ Cabe ao profissional médico desenvolver habilidades de comunica- ção que serão individualizadas a cada paciente, considerando-se a multiplicidade cultural, étnica e educacional de cada indivíduo, utilizando a entrevista médica como meio para obter informações clínicas, construir um relacionamento e educar o paciente. ${ }^{9,10}$

Para a pessoa que procura atendimento médico essa sistematização é desconhecida e o que ela mais solicita nesse encontro com o profissional médico é atenção às suas demandas e preocupações. Desse modo, o indivíduo tem necessidade de ser ouvido, de poder dizer ao médico tudo o que está sentindo ou lhe preocupando, sem importar-se com o tempo disponível para o atendimento ou a lógica interpretativa profissional de estabelecer limites e parâmetros para a elaboração da história clínica. ${ }^{11,12}$ Estudos têm demonstrado que a relação médico-paciente fica facilitada e ocorre com mais naturalidade à medida que o médico demonstra mais interesse e estimula o diálogo, ou seja, escuta o paciente, deixando-o falar livremente e respeitando sua ansiedade durante a consulta. ${ }^{13,14}$

O estabelecimento de uma adequada relação entre médico e paciente é a base para que o trabalho profissional se inicie e seja bem desenvolvido, visando oferecer aos indivíduos todos os elementos necessários para o atendimento de suas preocupações, seja na recuperação da saúde, nos aspectos preventivos ou de reabilitação. ${ }^{15,16}$ Diante disso, o objetivo deste estudo é conhecer algumas características relaciona- 
das ao atendimento médico nos ambulatórios do Hospital das Clínicas da Faculdade de Medicina de Ribeirão Preto da Universidade de São Paulo (HCFMRPUSP), no que diz respeito ao contato e ao diálogo entre paciente/família e corpo clínico.

\section{Metodología}

Trata-se de um estudo retrospectivo, observacional e descritivo desenvolvido durante o período de 01/08/2013 a 31/10/2013 nos ambulatórios de especialidades pediátricas do Hospital das Clínicas da Faculdade de Medicina de Ribeirão Preto - USP, para o qual foram convidadas a participar todas as mães de crianças que estavam aguardando atendimento médico. Os critérios de inclusão foram crianças na faixa etária do nascimento aos nove anos completos, que já haviam sido atendidas no mínimo uma vez no serviço e que estivessem aguardando atendimento nos ambulatórios das especialidades pediátricas do referido hospital. A todas as mães que concordaram em participar do estudo foi solicitado que assinassem um Termo de Consentimento Livre e Esclarecido e respondessem a um questionário, desenvolvido especificamente para esse propósito, que foi aplicado por duas pesquisadoras treinadas e do qual constavam perguntas relativas à criança (idade, sexo, tempo de acompanhamento na especialidade), à mãe (idade, grau de escolaridade e situação conjugal) e à consulta (relacionamento com o médico, entendimento das orientações, aspectos positivos e negativos das consultas e sugestões para melhoria do atendimento).

A amostra populacional deste estudo foi composta por 118 mães com mediana de idade de 32 anos, sendo $53,3 \%$ casadas e $22,8 \%$ solteiras, e com mediana de nível de escolaridade no segundo grau completo. Entre as crianças, 57,6\% eram do sexo femini- no e $42,3 \%$ masculino. Quanto ao tempo de seguimento nos ambulatórios a média foi de 2 anos e 1 mês e a mediana, de 12 meses. O estudo foi aprovado pelo Comitê de Ética em Pesquisa do Hospital das Clínicas da Faculdade de Medicina de Ribeirão Preto da Universidade de São Paulo, processo ${ }^{\circ}$ 118.145, de 09/10/2012.

\section{Resultados}

Todas as respostas obtidas pelos questionários foram agrupadas em categorias principais e encontram-se apresentadas nas tabelas de 1 a 4 . O uso da linguagem com terminologia médica $(50 \%)$ e a referência de que o médico não explica adequadamente $(19,5 \%)$ foram os aspectos que mais contribuíram para que a mãe não compreendesse corretamente o que o médico queria expressar durante a consulta. Por outro lado, a atenção dispensada ao paciente/família $(34,7 \%)$ e o exame clínico detalhado $(17,0 \%)$ foram os aspectos positivos mais citados, enquanto que a demora $(57,6 \%)$ e o fato de ser atendido por diferentes profissionais em cada consulta $(11,8 \%)$ foram relatados como aspectos negativos. Quando solicitadas a oferecerem sugestões que possam melhorar o atendimento, 38,1\% apontaram agilidade/rapidez e 12,7\% gostariam de receber dos médicos explicações mais simples.

Na Tabela 1 encontram-se relacionados os principais motivos, referidos pelas mães, que dificultam a comunicação durante o atendimento médico.

Nas Tabelas 2 e 3 encontram-se descritos, respectivamente, os aspectos considerados positivos e negativos relacionados ao atendimento.

As pessoas entrevistadas também foram solicitadas a oferecer sugestões para melhorar o atendimento e os resultados podem ser observados na Tabela 4.

Tabela 1. Aspectos relacionados ao não entendimento durante o atendimento médico. Ribeirão Preto 2013.

\begin{tabular}{lcc}
\hline & $\mathbf{n}$ & $\%$ \\
\hline Uso de terminologia técnica & 59 & 50,0 \\
Médico não explica adequadamente & 23 & 19,5 \\
Dificuldade de relacionamento com o médico & 9 & 7,6 \\
Não sabe perguntar para o médico & 9 & 7,6 \\
Médicos diferentes em cada consulta & 9 & 7,6 \\
\hline
\end{tabular}


Tabela 2. Aspectos positivos relacionados ao atendimento. Ribeirão Preto, 2013.

\begin{tabular}{lcc}
\hline & $\mathbf{n}$ & $\%$ \\
\hline Atenção dispensada ao paciente/família & 41 & 34,7 \\
Exame clínico detalhado & 20 & 17,0 \\
Educação dos profissionais & 17 & 14,4 \\
Paciência & 10 & 8,4 \\
Explicação sobre a doença & 10 & 8,4 \\
\hline
\end{tabular}

Tabela 3. Aspectos negativos relacionados ao atendimento. Ribeirão Preto, 2013.

\begin{tabular}{lcc}
\hline & $\mathbf{n}$ & $\%$ \\
\hline Demora & 68 & 57,6 \\
Diferentes profissionais em cada consulta & 14 & 11,8 \\
Falta de atenção & 8 & 6,8 \\
Divergência de informações & 8 & 6,8 \\
Má vontade do médico & 6 & 5,0 \\
\hline
\end{tabular}

Tabela 4. Sugestões dos acompanhantes para melhorar o atendimento. Ribeirão Preto, 2013.

\begin{tabular}{lcc}
\hline & $\mathbf{n}$ & $\%$ \\
\hline Agilidade/rapidez & 45 & 38,1 \\
Explicações mais simples & 15 & 12,7 \\
Agendar horários mais precisos & 12 & 10,1 \\
Atendimento pelo mesmo médico & 12 & 10,1 \\
Melhorar diálogo com o paciente/família & 9 & 7,6 \\
\hline
\end{tabular}

\section{Discussão}

Os resultados do presente estudo vão ao encontro do que foi observado por outros autores, corroborando o entendimento de que a comunicação deve contemplar a transmissão de informações, pensamentos e sentimentos que sejam satisfatoriamente recebidos e compreendidos por ambas as partes. ${ }^{17,18}$ Aqui, os aspectos referentes à comunicação entre médico e paciente/família ficaram evidentes nas respostas fornecidas pelas mães. 50\% delas atribuíram à termi- nologia utilizada pelo médico o aspecto que mais dificultou o entendimento das orientações durante a consulta, enquanto $19,5 \%$ referiram que o médico não explicou direito sobre o andamento e o resultado do atendimento. Destaque-se, portanto, a grande importância que a comunicação assume nesse contexto, permeando primariamente a relação entre médico e paciente/família. $\mathrm{O}$ encontro entre o agente (médico) e o paciente/família compreende um espectro dinâmico de elementos que exigem capacidade técnica, preceitos éticos, conhecimento, habilidades e sensibili- 
dade de ambas as partes, principalmente do profissional que assume papel ativo nessa relação. ${ }^{19,20} \mathrm{Em}$ sua essência a consulta médica geralmente se traduz como uma situação geradora de ansiedade, visto que o paciente, principalmente de classes sociais menos favorecidas, encontra mais dificuldades em relacionar-se utilizando esse tipo de comunicação formal. ${ }^{21}$

Alguns estudos tem evidenciado que os pacientes esperam do médico atenção, paciência para ouvir e disposição para conversar bastante, além da sinceridade quando abordarem questões relacionadas ao diagnóstico e tratamento. ${ }^{22,23}$ Em diversas situações o estilo de comunicação pode predominar sobre a qualidade técnica no transcorrer da consulta, principalmente quando o paciente participa da escolha do profissional médico que lhe prestará atendimento. ${ }^{24}$

Quando a comunicação entre as partes ocorre de maneira satisfatória, observa-se maior adesão ao tratamento, melhores resultados clínicos, redução de prescrições medicamentosas e, inclusive, diminuição de ações contra os médicos. ${ }^{25,26,27}$

Périco et al $2006^{28}$, estudando a relação médico-paciente em um hospital universitário observaram que a atenção dispensada pelo médico foi um fator muito destacado pelos pacientes, sendo que a conversa, o interesse e o fato de perguntar bastante acerca da criança foram considerados imprescindíveis para que os familiares se sentissem bem atendidos. Portanto, é possível afirmar que a satisfação do paciente/ família está diretamente relacionada com a quantidade e a qualidade das informações fornecidas pelo profissional sobre suas condições de saúde. ${ }^{29}$

Quanto aos aspectos considerados positivos durante $o$ atendimento, as entrevistadas destacaram a atenção dispensada pelo médico $(34,7 \%)$ e o detalhamento no exame clínico $(17,0 \%)$. Tais achados poderiam, embora aqui não se tratasse de um objetivo do estudo, fornecer indicativos sobre o grau de satisfação dos usuários, conforme também foi observado por Wanzer et al $2004^{30}$ e Brédart et al $2005^{31}$, quando constataram que a satisfação do paciente estava fortemente associada com a comunicação que ocorreu durante as consultas.

A demora, entendendo-se aqui o tempo de permanência na sala de espera, e a presença de diferentes profissionais médicos em cada consulta foram os fatores negativos mais referidos. De certo modo, esses fatores podem ser considerados inerentes a um serviço acadêmico de referência que, devido às suas especificidades, não tem a agilidade pretendida pelos usuários, visto que suas características de duplicidade de ações acadêmicas e assistenciais tornam particularizadas as relações entre usuários, gestores e prestadores de serviços. ${ }^{32}$

Aqui é importante destacar que em um hospital universitário de nível terciário como o HCFMRPUSP, recebe pacientes encaminhados de outros serviços de saúde, nem sempre existindo a vontade própria de escolha ou o desejo de ser atendido em tal lugar. Embora entre as classes mais populares seja disseminada a aura que envolve um hospital desse nível, talvez desejo íntimo de muitos pacientes, para outros, porém, não há possibilidades de escolha. Nessas situações, a relação médico-paciente acaba evoluindo para uma relação instituição-doença, configurando uma despersonalização ou perda de identidades pessoais, em virtude dos mecanismos e conceitos envolvidos nesse tipo de atendimento e nessas instituições ${ }^{33}$, onde os encontros são mais pontuais, objetivos, permeados pela variabilidade de personagens médicos diferentes, que tornam a relação assimétrica. ${ }^{34,35}$

Quando solicitado que fossem apontadas sugestões para melhorar o atendimento pode-se observar que a agilidade/rapidez $(38,1 \%)$, explicações mais simplificadas $(12,7 \%)$ e agendamento de horários $(10,1 \%)$ foram as respostas que mais se destacaram. Ainda aqui aparece a comunicação como um dos elementos considerados importantes para a melhoria do atendimento. Resultados semelhantes foram encontrados por Franco e Campos 1998, quando estudaram $\mathrm{o}$ atendimento ambulatorial de pediatria na cidade de Campinas (SP). No estudo, a demora e a consulta com médicos diferentes foram apontados pelos usuários como aspectos que deveriam ser modificados para melhoria do atendimento. ${ }^{36}$

Embora este estudo tenha sido realizado em um hospital universitário de referência e com uma amostra relativamente pequena de indivíduos seus resultados podem oferecer elementos para que sejam repensadas, pelo menos em parte, as características e os aspectos envolvidos na relação médico-paciente/família, considerando-se o ponto de vista da parte passiva dessa relação, cujos problemas de saúde demandam mais que atenção técnica e especializada, iniciando pelo contato humano que deve ser facilitado em todos os aspectos e passando pelo modo de comunicação e pelo acolhimento de que são merecedores todos os indivíduos. ${ }^{37}$

É indiscutível que as habilidades de comunicação são fundamentais para o estabelecimento de uma 
adequada relação médico-paciente e, embora esteja presente em muitos currículos de escolas médicas, esse treinamento geralmente ocorre nos períodos iniciais do curso e não encontra reforço nos estágios subsequentes ${ }^{38-40}$ Portanto, é desejável que essas habilidades sejam trabalhadas constantemente, visando seu aprimoramento e qualificação para o enfrentamento das inúmeras situações com que se deparam os médicos em suas atividades rotineiras.

\section{Referências}

1. King A, Hoppe RB. "Best Practice" for patient-centered communication: a narrative review. J Grad Med Educ 2013; 5: 38594.

2. Mead N, Bower P. Patient-centredness: a conceptual framework and review of the empirical literature. Soc Sci Med 2000; 51: 1087-110.

3. Silverman J, Kurtz S, Draper J. Skills for communicating with patients. London: Radcliffe Medical Press; 2005.

4. Ellison D. Building open communication for better patient care. Minn Med 2012; 35: 98-9.

5. Soar Filho EJ. A Interação médico paciente. Rev Assoc Med Bras. 1998; 44: 35-42.

6. Maguire P, Pitceathly A. Key communication skills and how to acquire them. BMJ 2002; 325: 697-700.

7. Pendleton D, Schofield T, Tate P, Havelock P. A Nova Consulta - Desenvolvendo a comunicação entre medico e paciente. Porto Alegre: Editora Artmed; 2011.

8. Suguimatsu LCF, Campos LFLCM, Geara LFM, Simões JC. A arte de ouvir o paciente. Rev Méd Resid. 2012; 14: 256-9.

9. Levinson W, Pizzo PA. Patient-physician communication: it's about time. JAMA 2011; 305: 1802-6.

10. Kroenke K. A practical and evidence-based approach to common symptoms: a narrative review. Ann Intern Med. 2014; 161: 579-86.

11. Sucupira AC. A importância do ensino da relação médicopaciente e das habilidades de comunicação na formação do profissional de saúde. Interface Comun Saúde Educ. 2007; 11: $619-35$

12. Porto $D$, Schierholt SR, Costa AM. Retratos da relação médico-paciente na atenção básica. Rev Bioet. 2012; 20: 288-99.

13. Manitta G. El malestar em la relación médico-paciente. Acta Bioeth.2005; 11: 85-94.

14. Morinaga CV, Konno SN. Aisawa RK, Vieira JE, Martins MA. Frases que resumem os atributos na relação médico-paciente. Rev Bras Educ Med. 2002; 26: 21-7.

15. Branda LA, Clèries $X$. Communication in the domain of health. Educ Med.2007; 10: 67-8.

16. van Dalen J, van den Eertwegh V. Communication in the initial paediatric consultation. Paediatr Respir Rev. 2013; 14:209-12.

17. Roter DL, Frankel RM. Hall JA, Sluyter D. The expression of emotion through nonverbal behavior in medical visits. Mechanisms and outcomes. J Gen Intern Med. 2006; 21 (suppl1): S2834.

18. Politi MC, Han PK, Col NF. Communicating the uncertain of harms and benefits of medical interventions. Med Decis Mak. 2007; 27: 681-95.
19. Grosseman S, Stoll C. O ensino-aprendizagem da relação médico-paciente: um estudo de caso com estudantes do último semestre do curso de medicina. Rev Bras Educ Méd. 2008; 32: 301-8.

20. Gabarra LM, Crepaldi MA. A comunicação médico-paciente pediátrico-família na perspectiva da criança. Psicol Argum. 2011; 29: 209-18.

21. Caprara A, Franco ALS. A relação paciente-médico: para uma humanização da prática médica. Cad Saúde Pública.1999; 15:847-54

22. Lemme AC, Noronha G, Resende JB. A satisfação do usuário em hospital universitário. Rev Saúde Pública.1991; 25: 41-6.

23. Jung HP, Home FV, Wensing M, Hearshaw H, Grol R. Wich aspects of general practioner's behavior determine patients' evaluation of care? Soc Sci Med. 1998; 47: 1077-87.

24. Buller KM, Buller BD. Physicians communication style and patient satisfaction. J Health Soc Behav. 1987; 28: 375-88.

25. Rao JK, Anderson LA, Inui TS, Frankel RM. Communication interventions make a difference in conversations between physicians and patients: a systematic review of the evidence. Med Care. 2007; 45: 340-9.

26. Clères $X$. La esencia de la comunicación em educación médica. Educ Med. 2010; 13: 25-31.

27. Street RL, Makoul G, Arora NK, Epstein RM. How does communication heal? Pathways linking clinician-patient communications to health outcomes. Patient Educ Couns. 2009; 74: 295-301.

28. Périco GV, Grosseman S, Robles ACC, Stoll C. Percepção de mães sobre a assistência prestada a seus filhos por estudantes de medicina da sétima fase: estudo de caso no Ambulatório de Pediatria de um Hospital Universitário. Rev Bras Educ Med. 2006; 30: 49-55

29. Patrick LD, Scrivens E, Charlton RHJ. Disability and patient satisfaction with medical care. Med Care. 1983; 21: 1063-75.

30. Wanzer MB, Nooth-Butterfield M, Gruber K. Perceptions of health care providers' communication: relationships between patient-centered communication and satisfaction. Health Commun. 2004; 16: 363-84.

31. Brédart A, Bouleuc C, Dolbeault S. Doctor-patient communication and satisfaction with care in oncology. Curr Opin Oncol. 2005; 17: 351-4.

32. Medici AC. Hospitais Universitários: passado, presente e futuro. Rev Assoc Med Bras. 2001; 47: 149-56.

33. Clavreul J. A Ordem Médica. São Paulo: Editora Brasiliense; 1983.

34. Fernandes JCL. A Quem interessa a relação médico-paciente? Cad Saúde Pública.1993; 9: 21-7.

35. Bendapudi NM, Berry LL, Frey KA, Parish JT, Rayburn WL. Patients' perspectives on ideal physician behaviors. Mayo Clin Proc. 2006; 81: 338-44.

36. Franco SC, Campos GWS. Avaliação da qualidade de atendimento ambulatorial em pediatria em um hospital universitário. Cad Saúde Pública.1998; 14: 61-70.

37. Ballester D. A Inclusão da perspectiva do paciente na consulta médica: um desafio na formação do médico. Rev Bras Educ Med. 2010; 34: 598-606.

38. Breuner CC, Moreno MA. Approaches to the difficult patient/ parent encounter. Pediatrics. 2011; 127: 163-9.

39. Wouda JC, van de Wiel HB. The communication competency of medical students, residents and consultants. Patient Educ Couns. 2012; 86: 57-62.

40. Karkowsky CE, Chazotte C. Simulation: improving communication with patients. Semin Perinatol. 2013; 37: 157-60. 